\title{
El problema de los valores éticos en la ciencia básica ${ }^{1}$
}

\author{
PILAR BELTRÁN ORENES \\ Universidad de La Coruña
}

\begin{abstract}
RESUMEN
Uno de los problemas centrales de la Ciencia hoy es la demanda social de responsabilidades éticas. Esta exigencia social procede del enorme impacto extracientifico de sus logros. La distinción epistemológica y metodológica entre Ciencia básica, aplicada y Tecnología suele incidir para delimitar las responsabilidades éticas de cada una. Así, la tendencia más extendida es aceptar criterios éticos para la actividad científica aplicada y el quehacer tecnologico, quedando al margen la valoración correspondiente a la Ciencia básica. En el presente estudio se examina la posibilidad y legitimidad de extender a la Ciencia bísica este tipo de valoraciones.
\end{abstract}

PALABRAS CLAVE

VALORES ETICOS-CIENCIA BÁSICA-CIENCIA APIICADA-TECNOLOCIA

ABSTRACT

One of the central problems of Science currently is the social demand for ethical responsibilities. This is causcd by the huge extrascientifical impact of Science's achievements. The epistemological and methodological distinction among basic Science, applied Science and Technology tends to determine the repestive attribution of ethical responsibilities. Hence, usually it is assumed that applied Science and Technoly can be ethically evaluated. But that is not the case of basic Science. This paper examines both the possibility and the legitimacy of extending to basic Science this kind of evaluations.

KEYWORnS

ETHICAL VALUES-BASIC SCIENCE-APPLIED SCIFNCE-TECHNCLOCIY

1 La versión preliminar de este trabajo fue presentada en las.Jornadas sobre Ciencia y Valores Éticos, Congreso organizado por la Facultad de Humanidades de la Universidad de La Coruña y celebrado los días 5 y 6 de marzo de 1998.

(1) Contrastes Revista Intenfisciplinar de Filosufita vol. III (1998), fD. 17-40. ISSN 1136-4076

Sección de Filosofia, Universidad de Málaga, Facultad de Filosofía y Letras

Campus de Teatinos, E-29071 Málaga (España) 


\section{PLANTEAMIENTO DE LA CUESTIÓN}

EN EL PRESENTE ESTUdIO SE BUSCA EL ESCLARECIMIENTO de algunos de los aspectos relacionados con la aplicación de valores éticos en el ámbito científico. La posibilidad de valorar éticamente el quehacer científico y sus resultados es uno de los problemas que presenta actualmente una mayor dificultad, por estar conectado en última instancia con temas tan relevantes como la libertad del científico. Este análisis se emprende sin intención de agotar la discusión sobre las cuestiones relativas a las posibles aplicaciones de códigos éticos a la Ciencia. La única tarea que se busca conscientemente es señalar algunos de los puntos conflictivos que plantea la inclusión en el ámbito científico de este tipo de consideraciones, y las posibles conexiones entre la Ciencia básica, la Ciencia aplicada y la Tecnología, nexos que pueden arrojar luz sobre la pertinencia o no de valoraciones éticas en estos tres ámbitos.

El planteamiento de fondo que motiva la reflexión es la separación habitual entre la Ciencia básica, por una parte, y la Ciencia aplicada y la Tecnología, por otra, en cuanto a consideraciones éticas de carácter interno y externo. Así, mientras que la Ciencia básica suele pasar con holgura las revisiones éticas relativas a valores internos (honradez del científico, búsqueda de la verdad, etc.), la Ciencia aplicada y la Tecnología suelen quedar exentas de este tipo de consideraciones, pues tanto el científico que orienta su investigación a la aplicación como el tecnólogo, parecen no tener que guiarse más que por la relación costes-beneficios. En otras palabras, un científico básico puede ser bueno y honrado por definición, mientras que el científico aplicado o el tecnólogo no recibe ninguno de estos calificativos, también por definición. En cuanto a las consideraciones éticas de carácter externo, ocurre precisamente lo contrario, mientras el científico aplicado y el tecnólogo deben calibrar con precisión las consecuencias de sus desarrollos, el científico básico queda exento de este tipo de consideraciones pues sus incrementos cognoscitivos no tienen consecuencias externas. Y sólo tendrá que responder en los casos en los que se produzcan aplicaciones científicas o cambios tecnológicos directos a causa de sus conquistas epistemológicas. Aquí el científico aplicado o el tecnólogo son los moralmente evaluables, mientras que los científicos básicos están exentos de este tipo de calificativos éticos.

Para conseguir una mejor inteleccción del problema, se examinarán ciertos elementos de conexión entre las distintas parcelas científicas y el ámbito tecnológico, según la clasificación antes mencionada: Ciencia básica, aplicada y Tecnología. Se intentan establecer aquí algunas vías que permitan esclarecer temas como la posibilidad y legitimidad de valores éticos en la Ciencia básica, lo que conlleva aclarar las relaciones con la Ciencia aplicada y la Tecnología, en cuyas actividades - científica aplicada y quehacer tecnológico-es más pa- 
tente la presencia de factores éticos, que en el ámbito de la actividad científica básica.

En este trabajo, además de las vertientes epistemológicas y metodológicas relativas a la distinción entre Ciencia básica, Ciencia aplicada y Tecnología, se propone un camino poco habitual en el campo de la Filosofía y la Metodología de la Ciencia, pero que empieza a ser usado por pensadores del prestigio de Nicholas Rescher, Ilkka Niiniluoto y Evandro Agazzi. Esta nueva vía consiste en resaltar dos facetas complementarias: por un lado, la caracterización de la actividad científica como una actividad humana más entre otras -como señalan Rescher ${ }^{2}$ y Agazzi ${ }^{3}$-y, por otra parte, la aceptación de la índole cultural y social de la actividad científica (todo ello sin que esto suponga olvidar que hay planos estrictamente filosóficos y metodológicos que diferencian las distintas parcelas científicas, tal como sostiene Niiniluoto ${ }^{4}$.

\section{COORDENADAS DEL PROBLEMA}

La enorme repercusión extracientífica que tienen actualmente los desarrollos de la Ciencia ${ }^{5}$ hace patente la necesidad de llevar a cabo una reflexión sobre la actividad científica que contemple los valores internos y externos que ésta comporta $^{6}$. Esta reflexión es urgente debido, por una parte, a la importancia de las consecuencias de la propia actividad científica - la faceta externa de la práctica científica- en ámbitos como el bioquímico o el correspondiente a la Biología molecular (en especial, los estudios referidos a Genética humana, que están poniendo en primera línea cuestiones tales como la elección del sexo de los futuros fetos, del color de ojos, etc.). Por otra parte, están también los aspectos internos acerca de las intenciones que guían a los científicos cuando llevan a cabo determinadas investigaciones (la posibilidad de crear nuevas vidas hu-

2 N. Rescher, The Limits of Science. Berkeley: University of California Press, 1984, pp. 208-215.

3 E. Agazzi, El bien, el mal y la ciencia. Las dimensiones éticas de la empresa científicotecnológica, tr. R. Queraltó. Madrid: Tecnos, 1996.

4 I. Niiniluoto, «Ciencia frente a Tecnología: ¿Diferencia o identidad?», Arbor, v. 620 , (1997), pp. 285-299.

5 P. Martínez-Freire, «Ciencia y Sociedad», Philosophica Malacitana, III (1990), p. 168. En este artículo, Martínez-Freire resalta la importancia que ha tenido el hecho de que hayan llegado al gran público informaciones referidas a la distribución de fondos por parte de los Estados, lo que activa las reclamaciones de responsabilidades éticas a la Ciencia y la Tecnología

6 Una primera aproximación en esta dirección fue el libro de L. Laudan, Science and Values. Berkeley: University of California Press, 1984. Su enfoque de los valores se reduciría al plano interno (en concreto, a los valores cognoscitivos o epistémicos), mientras que aquí se consideran también los factores externos. 
manas - $\mathrm{y}$, por qué no, vidas no humanas también- de manera arbitraria que se plantea en casos como la clonación).

Ahora bien, analizadas con detalle las cuestiones éticas que plantea la Ciencia, se advierte que no están motivadas sólo por aquellos campos científicos que tienen una incidencia más directa en la vida del ser humano (o, más ampliamente, en el ámbito de la Naturaleza en general), como son la Ciencia aplicada y la Tecnología. La ampliación a otros casos -los ámbitos científicos considerados como puramente teóricos- responde a las relaciones existentes entre la Ciencia básica, la aplicada y la Tecnología, en cuanto a que, en muchos casos, las dos últimas realizan con éxito sus trabajos cuando usan con efectividad el soporte teórico que requieren sus desarrollos. También se produce el fenómeno contrario y, ante las demandas de la Ciencia aplicada y la Tecnología, la Ciencia básica pone a trabajar su maquinaria para conseguir el ansiado objetivo del conocimiento por el conocimiento, planteamiento que, en estos casos, no responde a razones puramente básicas. Así, parece necesario extender al ámbito de la Ciencia básica el tipo de consideraciones propias de la Ética de la Ciencia, incluyendo aquel plano que no se circunscribe al ámbito interno.

Entre los planteamientos que surgen a raíz del problema acerca de la necesidad de aplicar criterios de tipo ético a la actividad científica figuran dos posiciones particularmente relevantes y extremas: por un lado, la insistencia en la plena autonomía y la autoridad de la Ciencia, que lleva a algunos científicos a exigir el Laissez faire (el dejar hacer); y, por otro lado, el énfasis en la relevancia social de la Ciencia, sobre todo la Ciencia aplicada y su proyección tecnológica, que, en algunos casos, conduce al extremo opuesto, esto es, a la Panregulación de la Ciencia ${ }^{7}$. Así, la reflexión ética sobre el edificio científi$\operatorname{co}^{8}$, con los tres grandes ámbitos que la componen: la Ciencia básica, la aplicada y la Tecnología, se mueve entre la dinámica interna de la actividad científica y la faceta externa de sus consecuencias?.

Estos dos planteamientos extremos -el dejar hacer y la hiperregulaciónreflejan, a mi entender, las dos posiciones más radicales. Así, desde la primera postura se reivindica la autonomía de la Ciencia alegando su bondad intrínseca, y en ella se emplean el tipo de argumentos que se esgrimen cuando se pretende dejar a la Ciencia básica al margen de consideraciones éticas: la Ciencia básica busca como fin primero alcanzar el conocimiento por el conocimiento,

7 N. Rescher, Razón y valores en la era científico-tecnológica. Libro Inédito, capítulo 8.

8 Empleo aquí la expresión «edificio científico» en su sentido más amplio y laxo. Desde esta perspectiva, caerán bajo esta denominación tanto los desarrollos relacionados con la Ciencia estrictamente como los que se llevan a cabo en el ámbito tecnológico.

9 Cabe recordar aquí que los valores éticos relacionados con el edificio científico pueden ser de carácter interno (honradez y veracidad, por ejemplo) y de índole externa (usos legítimos o ilegítimos del conocimiento y de los resultados científicos y tecnológicos, entre otros). 
lo que es considerado bueno en sí mismo. Los científicos básicos, guiados por ese fin y asegurando que el conocimiento por el conocimiento no tiene ninguna repercusión externa que pueda ser nociva -saber nunca es malo a priori-, quedarían exentos de una evaluación desde el punto de vista ético externo, esto es, en cuanto a las consecuencias de su actividad. De esta manera, la falta de aplicación directa de los conocimientos básicos es el motivo alegado usualmente para liberar a la Ciencia básica de las consideraciones éticas. En ese caso, se admite que el quehacer científico es autorregulador: consigue corregir, por mecanismos autónomos y específicos de la Ciencia, las posibles desviaciones, tanto en los procesos de adquisición de conocimiento como en la actuación del propio investigador (quien, además de perseguir el fin de la Ciencia, puede incluir sus propias finalidades personales en el proceso investigador). Cabe añadir que la extensión de este tipo de argumentos a la Ciencia aplicada y a la Tecnología es una cuestión más difícil, debido a su patente incidencia externa, que pone de relieve su responsabilidad social.

Por otra parte, la postura que opta por la Panregulación dirige la mirada hacia el otro extremo del problema e incide que la Ciencia aplicada y la Tecnología tienen que responder acerca de las consecuencias de sus desarrollos. $\mathrm{La}$ Ciencia básica, por su parte, debe atender también a las consideraciones éticas, ya que la adquisición de conocimiento también concede poder, y el poder cabe ser interpretado como una consecuencia. La argumentación en este caso está orientada en sentido contrario a la empleada en las posiciones que propugnan la total autonomía; es decir, se parte de las consecuencias que tiene la intencionalidad del científico -tanto básico como aplicado- y del tecnólogo sobre el proceso mismo de investigación. Así pues, hay que poner en tela de juicio incluso la bondad intrínseca del propio proceso.

Aunque ambas posturas parecen atender a las vertientes internas y externas de la valoración ética, usan, en última instancia, argumentos que parten de uno de estos dos planos para apoyar sus argumentaciones. La primera opción -Laissez faire - atiende fundamentalmente a los elementos internos de la investigación científica - la autorregulación-con vistas a realizar la valoración ética: justifica, así, el plano externo de las consecuencias desde el interno y deja a la Ciencia básica claramente al margen de este tipo de consideraciones éticas. La segunda -la hiperregulación- extiende a todos los ámbitos de la Ciencia las valoraciones éticas -en concreto, incluye al básico-; ahora bien, establece la necesidad de control ético en virtud de las consecuencias que tienen todas estas actividades, incluyendo la actividad científica pura (el saber concede poder), esto es, justifica la necesidad de control desde la perspectiva que marcan los factores externos.

La presencia constante de estas dos coordenadas en todo quehacer científico -las perspectivas interna y externa- $y$ la facilidad con la que se ignora 
alguna de ellas, alegando argumentos sesgados como los anteriores, explica que algunos autores, como los partidarios del Neopositivismo lógico, hayan visto la Ciencia -en sintonía con lo que había señalado $\mathrm{M}$. Weber 10 - como una empresa «libre de valores» (value free). Desde este planteamiento, la Ciencia debe ser entendida primordialmente como lenguaje, estructura y conocimiento, dotados de un estatuto propio al margen de cualquier valor ético. Para los defensores de este enfoque, la Ciencia está de suyo bien orientada: el conocimiento es un bien en sí mismo, por eso proponen un estricto Laissez faire. Al resaltar el conocimiento y dejar olvidada la variedad de consecuencias que se derivan de la aplicación científica y de la Tecnología, estos autores dejan fuera del quehacer científico todo valor que no sea interno (normalmente cognoscitivo, o relacionado directamente con este ámbito).

En el polo opuesto se sitúan concepciones actuales de la Ciencia dentro de la Sociología del Conocimiento, donde los valores externos poseen un lugar fundamental y definitivo en el quehacer científico, pues condicionan la dinámica interna de la Ciencia. Para este tipo de posiciones la pista suelen ser las consecuencias que tienen la Ciencia -en especial, la aplicada-y la Tecnología, que son vistas como nocivas. Desde esta posición se llega a dar una explicación de la actividad científica de carácter exclusivamente extrínseco, es decir, todo desarrollo científico está motivado por alguna razón externa, que lo modula y condiciona (cuando no, lo determina) ${ }^{\prime \prime}$. Por este motivo, resulta natural su pretensión de establecer un control externo sobre la investigación científica, actitud que ataca directamente la libertad del científico (lo que, de alguna manera, implica una Panregulación).

Sin llegar a adoptar posturas extremas respecto a la relevancia para la Ciencia y la Tecnología de los factores externos, la mayor parte de las reflexiones filosóficas actuales reconocen un papel a los elementos extrínsecos, aunque se le suela atribuir un alcance más limitado que el propuesto por las posiciones sociologistas. Ahora bien, la pertinencia de los valores éticos en la reflexión sobre la Ciencia y la propia labor científica de investigación como valores en liza en la actividad científica -en especial, los de carácter extrínseco-suele limitarse a aquellos planos que tocan de lleno y de manera inmediata la vida del ser humano. Entre estos ámbitos se encuentran la Ciencia aplicada y la Tecnología, de modo que dejan normalmente al margen a la Ciencia básica, a la que no se le aplican estos patrones, porque en lo concerniente a sus

10 M. Weber, The Methodology of the Social Science, E. A. Shils y H. A. Finch (eds.). Glencoe: The Free Press, 1949.

11 La «completa politización de la Ciencia» a la que conduce, según Radnitzky, la Teoría de la Finalización, es analizada por W. J. González en «Progreso científico, autonomía de la Ciencia y realismo», Arbor, 135 (1990), pp. 101-102. 
consecuencias, quedan libres de «sospecha» al no ser éstas directamente empíricas y porque se supone, en contra de lo que defienden los sociologistas del conocimiento, que el científico cumple satisfactoriamente los valores éticos de carácter interno: es honrado, veraz, etc.

Así pues, es en los ámbitos de la Ciencia aplicada y de la Tecnología en los que se suelen demandar las responsabilidades éticas. Esta demanda tiene lugar de manera habitual, cuando la puesta en práctica de los progresos científicos no arrojan los resultados esperados o cuando son usados para fines distintos de los que habían sido planeados en un principio. La demanda de responsabilidades suele recaer en la Ciencia aplicada y en la Tecnología, que son las parcelas que, en última instancia, ofrecen los resultados más visibles y son también los ámbitos aparentemente más inteligibles para el conjunto de la sociedad desde el punto de vista de la familiaridad con la que llegamos a tratarlos (por ejemplo, millones de seres humanos hemos usado cotidianamente los aerosoles que dañaban la capa de ozono).

Cabría cuestionar, sin embargo, si todas esas aplicaciones no resultan incluso más ininteligibles que los resultados ofrecidos por la Ciencia básica, puesto que, además de sus aportaciones aplicadas y tecnológicas, incluyen una gran cantidad de conocimientos teóricos 12 (en el ejemplo anterior de los aerosoles, sin duda, un porcentaje elevadísimo de los usuarios desconocemos, casi por completo, cuáles son los fundamentos teóricos, además de los prácti$\cos$ ). La ignorancia acerca del funcionamiento y las bases teóricas como puede ser el proceso de combustión, puede llevar a desconocer las consecuencias nocivas -éticamente reprobables-que incluso nosotros mismos causamos con su uso indiscriminado, lo cual constituye un hecho calificable desde el punto de vista ético.

También cabría resaltar, como elemento influyente en toda esta cuestión, la gran diversificación actual del quehacer científico y tecnológico. La creciente especialización de la Ciencia y la Tecnología plantea la necesidad y utilidad de la diferenciación epistemológica y metodológica entre la Ciencia básica, la Ciencia aplicada y la Tecnología ${ }^{13}$. Ahora bien, si esta distinción resulta pertinente en el plano epistemológico y en el metodológico no ocurre lo mismo -a mi juicio- en el nivel correspondiente a la reflexión ética. En este último ámbito, la tripartición puede conducir, por ejemplo, a que determinadas áreas del campo tecnológico intenten buscar un subterfugio para escapar de la

12 cf. M. Sintonen, «Basic and applied sciences: Can the distinction (still) be drawn?», Science Studies, 2 (1990), p. 30.

13 I. Niiniluoto, «The Aim and Structure of Applied Research», Erkenntnis, v. 38 (1993), pp. 1-21, así como Is Science Progressive?. Dordrecht: Reidel, 1984. Cf. M. Sintonen, «Basic and Applied Sciences: Can the distinction (still) be drawn?», pp. 23-31. 
consideración ética que demanda la sociedad en el terreno del apoyo teórico en la Ciencia básica y de ésta en la Tecnología para conseguir sus progresos cognoscitivos. Esto es, existe la posibilidad real de que los científicos aplicados y los tecnólogos pretendan eludir las consecuencias de su actividad alegando que sus diseños y artefactos se ven conducidos sólo por factores éticos de carácter interno (honestidad, fiabilidad, etc.). Cabe también que, desde la Ciencia básica y en aras a su teoricidad, se puede estar cayendo en la tentación de pensar que su actividad está completamente al margen de consideraciones de tipo ético, lo cual no parece responder a la realidad.

\section{Ciencia básica, Ciencia aplicada y Tecnología}

Para aclarar la posibilidad de valoraciones éticas en la Ciencia básica no sólo internas $-y$, dentro de las internas, que no sean únicamente positivas-el primer paso a dar lo constituye la delimitación lo más precisa posible de las parcelas científicas y tecnológicas, atendiendo principalmente a las perspectivas epistemológicas y metodológicas. Con esta finalidad, se presentan y estudian las diferencias filosóficas (en especial, epistemológicas) y metodológicas que se dan entre la Ciencia básica, la Ciencia aplicada y la Tecnología. Posteriormente, se establecerán los distintos puntos de conexión que las unen. Se comienza, por tanto, con las diferencias que las separan.

Cabe afirmar que no hay un criterio uniforme de separación entre estas tres parcelas, pues existen actualmente diferentes propuestas que tienden a diluir la frontera entre la Ciencia y la Tecnología, como la presentada por Bruno Latour con su concepto Tecnociencia ${ }^{14}$, que apunta a la disolución de esas fronteras intelectuales. O si se prefiere las posiciones que siguen el enfoque constructivista, como las desarrolladas por Wiebe Bijker y John Law15, que buscan hacer desaparecer la línea divisoria entre la Ciencia y la Sociedad con la propuesta de la Sociotecnología. Ahora bien, como señala Niinilouto, tales análisis son una muestra del esfuerzo por intentar fusionar lo que en principio se presenta separado 16 .

En lo relativo a la fusión de la Ciencia y la Tecnología en un sólo concepto -Tecnociencia-, resulta claro - a mi juicio- que, tanto por sus objetivos y contenidos como por su Metodología, la Ciencia se diferencia conceptualmente de la Tecnología. La Ciencia reúne una serie de caracteres que le son propios: «i) es un tipo de conocimiento exhaustivo (más riguroso, por tanto, que el conocimiento

14 B. Latour, Science in Action. Milton Keynes: Open University Press, 1987, p. 29.

15 W. E. Bijker y J. Law, Shaping Technology/Building Society: Studies in Sociotechnological Change. Cambridge: The MIT Press, 1992.

16. I. Niiniluoto, «Ciencia frente a Tecnología: ¿Diferencia o identidad?», pp. 286-287. 
ordinario); ii) consiste en una actividad portadora de un método (normalmente deductivo); iii) posee un lenguaje específico (dotado de términos bien precisos); iv) aparece como una realidad dinámica (de carácter autocorrector, que busca incrementar los niveles de verosimilitud); v) posee una realidad propia, que surge de la acción social y está dotada de una serie de notas constitutivas que la distinguen de otras actividades, por sus presupuestos, contenidos y límites; vi) cuenta con fines -generalmente, cognoscitivos--, a los que se encamina su labor de investigación; $y$, vii) es susceptible de valoraciones éticas que atañen al proceso mismo de indagación (honradez, fiabilidad) y a su nexo con el resto de las actividades de la vida humanas 17

La Tecnología, por su parte, reúne una serie de requisitos: «a) consta de un lenguaje propio, que atiende a factores internos al proceso (diseño, eficacia, eficiencia) y a elementos externos (sociales, económicos, políticos, culturales, etc.); b) la estructura de los sistemas tecnológicos se encuentra articulada sobre la base de su operatividad, pues ha de servir para guiar la actividad creativa transformadora que realiza el sujeto humano sobre la Naturaleza (o, en su caso sobre la realidad social); c) el conocimiento específico del quehacer tecnológico (el know how) es instrumental e innovador: busca la intervención sobre un ámbito real, para su dominio y utilización al servicio de los agentes humanos y la Sociedad; d) el método seguido está modelado por una argumentación imperativo-hipotética, de modo que los fines buscados son los que hacen razonables (o no) a los medios encaminados a alcanzarlos; e) todo el proceso tecnológico está directamente influido por valores, tanto internos [...] como externos [...], que condicionan la viabilidad de la posible Tecnología a seguir y sus alternativas; $y$ f) la realidad misma del quehacer tecnológico se sustenta sobre acciones humanas sociales, dotadas de intencionalidad y encaminadas a la transformación de la realidad circundante» 18 .

La distinción entre Ciencia y Tecnología parece pertinente desde perspectivas epistemológicas y metodológicas, pero no es nítida, tal como remarca Niiniluoto ${ }^{19}$, así como tampoco resulta evidente la separación entre Ciencia básica y Ciencia aplicada. Ahora bien, sí cabe una distinción entre ellas, de modo que la Ciencia básica es, en principio, «aquella actividad de la comunidad científica que produce un conocimiento científico mediante el método científico» 20 , mientras que la Ciencia aplicada desarrolla aquella actividad que re-

17 W. J. González, «Progreso científico e innovación tecnológica: la 'Tecnociencia' y el problema de las relaciones entre Filosofía de la Ciencia y Filosofía de la Tecnologían, Arbor, 620 (1997), p. 265.

18 lbid., pp. 265-266.

19 I. Niiniluoto, «Ciencia frente a Tecnología: ¿Diferencia o identidad?», p. 287.

20 I. Niiniluoto, «The Aim and Structure of Applied Research», Erkenntnis, 38 (1993), p. 3. 
quiere una aplicabilidad práctica, de manera que, junto con el conocimiento, necesita las utilidades de «simplicidad» $y$ «capacidad de manejabilidad» 21 . A diferencia de las dos anteriores, la Tecnología se centra en el diseño de artefactos y su función primordial consiste en transformar la realidad en lugar de buscar conocimiento o conocimiento aplicable22.

Cuando la Ciencia se orienta hacia fines específicos, en lugar de buscar meramente el conocimiento, se torna Ciencia aplicada. En ella se busca la efectividad del conocimiento para un quehacer humano concreto, de modo que el conocimiento se convierte en un instrumento para el quehacer humano en el tiempo. Así, la Ciencia aplicada está entre los polos más alejados: la Ciencia básica y la Tecnología. Se admite, entonces, de manera tácita que la Ciencia aplicada es, en algunos casos, el puente entre la Ciencia básica y la Tecnología, pudiendo esta última ser considerada como Ciencia aplicada en ocasiones muy concretas 23 .

Junto a la distinción conceptual -y relacionada directamente con ella-, está el problema, de carácter pragmático, de la ausencia de lindes entre los cada día más numerosos ámbitos que se están generando en el campo científico, ámbitos que permanecen conectados. Este incremento, tanto científico como tecnológico, está provocando una serie de intentos de fundamentación teórica para cada uno de los planos afectados. Las distinciones entre la Ciencia básica, la aplicada y la Tecnología, como la que presenta Agazzi 24 , o la ya citada de Niiniluoto, no hacen sino reflejar la necesidad teórica de separación entre las actividades de carácter científico y las de tipo tecnológico, atendiendo a que son actividades humanas con distinto objeto y método (a las que frecuentemente se tiende a tratar-por los partidarios de la Tecnociencia-como actividades con objeto y métodos esencialmente idénticos).

En su clasificación del sistema científico, Agazzi se basa en varios puntos para la distinción entre Ciencia y Técnica: i) mientras la Ciencia tiene como función específica la adquisición del conocimiento (la ampliación de información), la Técnica se ocupa de la realización de ciertos procedimientos o productos (el hacer); ii) la Ciencia busca la verdad, mientras que la Técnica ejecuta algo útil25. A juicio de Agazzi, estas dos diferencias básicas no llegan a invalidar los distintos puntos que las unen: por un lado, hay una necesidad de tener artefactos técnicos para conseguir las metas científicas; $y$, por otro, existe una aplicación de los conocimientos científicos en el ámbito tecnológico26.

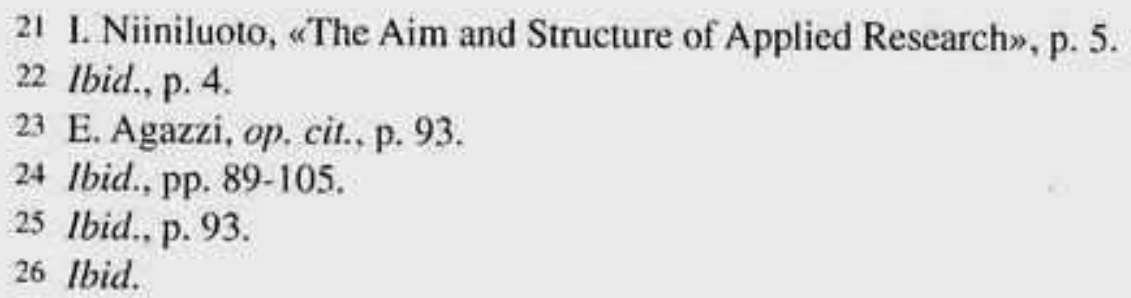


Esta última relación entre Ciencia y Técnica hace pertinente, a su entender, otra distinción ulterior entre Técnica y Tecnología, pasando a ser la «Tecnología» la parte de la «Técnica» que lleva a cabo la aplicación de los conocimientos teóricos al terreno práctico; para ello construye aparatos diseñados con una finalidad práctica directa ${ }^{27}$. La Tecnología es, de este modo, una Ciencia aplicada 28 .

Ahora bien, esta distinción tan pertinente desde los puntos de vista epistemológico y metodológico, no se presenta tan nítida -a mi entender-desde la perspectiva ética. Porque, actualmente, se suceden rápidos progresos, que tienen como correlato que nuevos instrumentos se estén poniendo en manos de un gran número de seres humanos y, en algunos casos, conllevan consecuencias que los tecnólogos no están dispuestos a admitir, alegando que sólo corresponden a las consecuencias del uso y no tienen nada que ver con la bondad del diseño de su artefacto 29 . De esta manera eluden posibles responsabilidades externas, alegando las impecables intenciones que, desde el punto de vista ético, les llevaron a su diseño y construcción. Esto es lo que ocurre claramente, a mi juico, en el caso de los científicos teóricos cuando se les reclaman responsabilidades. Estos últimos consideran que su labor sólo se encamina a la búsqueda del conocimiento, sin ninguna finalidad práctica, por lo que sobran las consideraciones éticas de carácter externo sobre su trabajo -las relativas a las consecuencias- $y$, aun más, puesto que las de carácter interno les son inherentes - es decir, ocurre, de hecho, la bondad entre los científicos, dentro de sus centros de investigación- redundan las revisiones éticas de cualquier tipo. Y, en el caso extremo en el que se dé un científico cuyo comportamiento sea éticamente incorrecto, será la propia comunidad científica quien se encargue de neutralizarlo.

Normalmente la sociedad, en general, tiende a traspasar todas las responsabilidades de la empresa científico-tecnológica a quienes hacen un uso de los conocimientos obtenidos, sean éstos de la Naturaleza o de la Sociedad. Así, la tendencia es hacer recaer las responsabilidades éticas sobre los tecnólogos o, de manera más abstracta, en la Tecnología y, a lo sumo, en la Ciencia aplicada, pero no en la Ciencia básica. Ahora bien, el hecho de que las distancias entre la Ciencia básica, la aplicada y la Tecnología (o, simplemente, entre la Ciencia y la Tecnología) puedan ser establecidas sólo en los planos epistemológicos y metodológicos, debería ser, a mi entender, tenida más en cuenta a la hora de llevar a cabo una reflexión sobre las distintas repercusiones éticas de la labor

27 Ibid., pp. 95-99.

28 Ibid., p. 102.

29 Según afirma Niiniluoto, los tecnólogos no se pueden desentender de los usos que se hagan de los artefactos que construyen. I. Niiniluoto, «Los límites de la Tecnología», Arbor, 620 (1997), p. 405. 
científica. Tal como señala Martínez-Freire, "teóricamente cabe hablar de un conocimiento por el propio conocimiento, pero en la práctica en nuestros días son cada vez más escasos los científicos 'puros'. Podemos librar de responsabilidad a los científicos básicos, en especial a los lógicos y matemáticos, pero resulta difícil eximir de responsabilidad a físicos y químicos teóricos, resultando indudable la responsabilidad de los tecnólogos»30. Martínez-Freire resalta, de nuevo, uno de los elementos fundamentales para el problema: aunque se pueda establecer la distinción teórica, las distintas parcelas de la Ciencia y de la Tecnología se presentan cada día más entrelazadas en la práctica, lo que hace que los científicos puros escaseen y que la posibilidad de aplicar intenciones y consecuencias a toda actividad científica se muestre plausible.

En cuanto a la propuesta sobre las posibles conexiones indisolubles entre la Sociedad y la Tecnología -la segunda objeción a la separación de los conceptos de Ciencia y de Tecnología, asimilándolos ambos al de Sociedad, la propuesta sociotecnológica- habría que indicar, como resalta Niiniluoto ${ }^{31}$, que es conveniente separar «las propiedades objetivas de un artefacto y los criterios de valor en su evaluación»32. Así, no se debe confundir el valor instrumental (su efectividad o no) con el incremento del dominio de la libertad humana que supone la efectividad de un artefacto. Esta última consecuencia -la ampliación del dominio de la libertad humana-sí es cuestionable desde el punto de vista ético, pues puede dar lugar tanto a un buen uso como a uno malo ${ }^{33}$. Por esto, no es viable la disolución apuntada por la Sociotecnología, en cuanto a la imposibilidad de hacer responsables a los tecnólogos, alegando que los artefactos que construyen no son sino los reclamados por determinados grupos sociales, sino que por el contrario, a mi entender, sí se les puede reclamar responsabilidades acerca de los usos de los artefactos que han construido. $\mathrm{El}$ indudable impacto social de los artefactos los pone claramente en primera línea en cuanto a la posibilidad de elaborar juicios éticos sobre ellos, y no los deja al margen de consideraciones éticas externas el argumento de ser objetos que, en muchos casos, son demandados por esa misma sociedad sobre la que se extienden sus consecuencias.

Así pues, los rasgos señalados de carácter epistemológico y metodológico permiten la distinción de los dominios respectivos de la Ciencia básica, la Ciencia aplicada y la Tecnología. Por otra parte, se han apuntado las relaciones limitadas entre la Tecnología y la Sociedad. Esta aproximación de fondo permite pasar al siguiente apartado donde se llevará a cabo un análisis desde el

30 P. Martínez-Freire, loc. cit, p. 171.

31 I. Niiniluoto, «Los límites de la Tecnología», pp. 404-408.

32 Ibid., pp. 404.

33 I. Niiniluolo, «Los límites de la Tecnología», pp. 404-405. 
punto de vista antropológico cultural, para ver en qué medida las distinciones epistemológicas y metodológicas que son necesarias para la distinción entre los distintos ámbitos de la actividad científica y tecnológica, pueden perder parte de su vigencia cuando se trata de lo relacionado con las consideraciones éticas.

\section{iv. Cultura, Etica y Ciencia}

Para poder establecer los canales de flujo a través de los que circulen los valores éticos que se pueden aplicar a toda la actividad científica -igual que los valores de verdad o falsedad dentro de una teoría-, es necesario completar el cuadro teórico que sirve de marco para los nexos con la Etica de la Ciencia, tanto la básica como la aplicada, y la Tecnología. En este apartado se resalta, pues, el punto principal que las tres tienen en común: el ser o estar relacionadas con actividades humanas. Las tres comparten así una dimensión práctica de carácter libre y autónomo, cuyo punto de partida es el sujeto humano y cuyo contexto es socio-cultural. Las tres tienen vínculos con la cultura humana, entendida ésta en su acepción más genérica desde el punto de vista antropológico cultural.

Esta última apreciación no resulta ociosa ya que, aún en nuestros días, sigue imperando en gran parte de la sociedad -al menos la occidental-la identificación del término cultura con la denominada «cultura del ocio», reduciendo así el ámbito de la cultura a un plano periférico e incidental (entretenimiento, diversión, ...), de modo que pasa por alto buena parte de los conocimientos que, gracias a los estudios antropológicos actuales, se han ido asentando con todo derecho.

Dentro del ámbito filosófico y metodológico no es tampoco redundante llevar a cabo esta distinción. Un autor de gran relevancia dentro de este ámbito, E. Agazzi, parece ser uno de los que incide en esta confusión, como se puede inferir de su clasificación de los distintos sistemas que constituyen el entramado de vías de intercambio y comunicación de la información a nivel mundial ${ }^{34}$. A su juicio, el sistema cultural, dentro de una sociedad concreta, es uno más entre los sistemas humanos como el político, el religioso, el moral y/ o ético, etc. Esto parece indicar, aunque ciertamente Agazzi no lo afirma, que los sistemas: militar, ético y/o moral, religioso, económico y político, entre otros sistemas según su esquema, se presentan separados de la cultura, esto es, no constituyen cultura, por lo que parece lógico inferir que cuando Agazzi habla de sistema cultural, piensa en la «cultura del ocio». 
La clasificación sistemática llevada a cabo por Agazzi es, sin duda, de gran valía a la hora de reconocer las distintas influencias mutuas de los distintos ámbitos y niveles que operan en el quehacer humano informativo. Ahora bien, a mi entender, falla en su consideración de lo que es el sistema cultural de un determinada sociedad. Su separación de los sistemas como el político, militar y ético está bastante lejos de ser una correcta interpretación desde el punto de vista antropológico cultural. Porque, desde esta perspectiva, la cultura está constituida, precisamente, por todos esos otros sistemas que Agazzi considera como separados del cultural (político, militar, religioso, etc.).

Esta deficiencia de su análisis en cuanto al lugar de la cultura, no invalida el resto de su enfoque. A este respecto, tal como ya se apuntó, Agazzi es uno de los autores que identifica con acierto la actividad científica como una actividad humana más entre todas las demás. Ahora bien, su visión parcial de la noción de cultura dificulta, a mi entender, la posibilidad de la conexión adecuada entre la Ciencia -tanto básica como aplicada y, en consecuencia, también la Tecnología- y la Ética, puesto que estos dos sistemas (el científico y el ético) quedan separados en su estudio y sin un marco común. Este marco, a mi entender, debería ser el cultural. Y ésta es una dificultad que no logra salvar el planteamiento presentado por Agazzi, que deja a la actividad científica como una actividad humana distinta de las restantes actividades humanas y también distinta de las acciones humanas instintivas. De hecho, en la propuesta de Agazzi existirían tantos tipos de actividades humanas como sistemas conforman el campo de acción humano, lo que implicaría una distinción en cuanto al grado en el que verse afectados por la reflexión ética cada uno de los tipos de actividad.

Dejando a un lado esta concepción de cultura empleada por Agazzi, y adoptando nociones más ajustadas a los planteamientos antropológicos culturales, resulta bastante claro que, en lo relativo al ámbito ético y científico, la parcela a la cual pertenecen este tipo de manifestaciones (por ejemplo, los juicios éticos y los enunciados científicos), forma parte del complejo que distingue a los seres humanos de otros individuos. En este sentido, se sitúa en el contex to de la cultura: es algo creado o, a lo sumo, heredado culturalmente, y no de la natura, pues no es algo recibido genéticamente (o meramente «ya dado»).

En esta dirección se encuentra la definición de cultura propuesta por el antropólogo E. B. Tylor, quien, a finales del siglo XIX, escribía que cultura es «todo complejo que incluye conocimientos, creencias, arte, leyes, moral, costumbres y cualquier otra capacidad y hábitos adquiridos por el hombre en cuanto miembro de una sociedad»35. De esta manera, las manifestaciones que conforman la cultura, entre las que se encuentran las consideraciones éticas y los

35 E. B. Tylor, Primitive culture, 2 vols., London: John Murray, 1871, p. 1. 
logros científicos, se definen como aquella parte del ser humano que es adquirida, no heredada genéticamente 36 .

Según la definición de Tylor, la Ciencia es un quehacer humano cultural, pues es una parcela en la que se trata con «información generada por aprendizaje individual (descubrimiento, invención) y transmitida por aprendizaje social»37. En este sentido, la Ciencia cae fuera de los límites de lo que es por natura ${ }^{38}$. Además, la Ciencia es un tipo de actividad humana que se encamina, fundamentalmente, al conocimiento preciso de la Naturaleza y de la Sociedad, actividad en la que imperan valores internos a la Ciencia como el rechazo a lo falso: la «abominación de la falsificación de datos» ${ }^{39}$, no puede ser entendida a la manera en que lo es el conocimiento espontáneo (el que los griegos identificaban con la opinión o «doxa» -concepto que podría ser paralelo al actual «sabiduría popular»-, y que suele surgir, de manera espontánea, de la interacción directa entre el ser humano y la Naturaleza). En la medida en que no es espontánea, la Ciencia es una actividad conscientemente querida: es voluntaria, de modo que está dotada de una intencionalidad 40 -la que corresponde al sujeto que la lleva a cabo-; se encuentra sometida, por tanto, al enjuiciamiento ético como cualquier otra actividad humana libre.

$\mathrm{Al}$ ser la Ciencia un elemento cultural ligado indisolublemente a las creaciones propias del ser humano, tiene una repercusión directa sobre el sujeto de experiencias en que consiste el ser humano ${ }^{41}$. Porque, en consonancia con la definición de Tylor, hacer cultura -y, con ella, Ciencia y Tecnología- no es otra cosa que encaminarnos a la dominación del medio natural que, en principio, nos es hostil; es hacernos un hueco en la Naturaleza (o bien adaptándonos o bien adaptándola) y crearnos una parcela distinta a la natural (porque se produce siempre una acomodación al medio o del medio), según nuestros propios deseos y, sobre todo, necesidades. La Ciencia -y, sobre todo, la Tecnología-, tiene entre sus metas la de intentar dominar el medio, para adaptarlo a nuestras

36 J. Mosterín, Filosofia de la cultura. Madrid: Alianza Editorial, 1994, pp. 17-18.

37 Ibid., p. 111.

38 En esta dirección se mueve N. Rescher cuando señala que la Ciencia es nuestra Ciencia, N. Rescher, «Nuestra Ciencia en tanto que nuestra», Daimon, 6 (1993), pp. 1-9.

39 J. Mosterín, op. cit., p. 112.

40 Se asume aquí que hay una distinción entre «intención» e «intencionalidad»; la primera no es observable, mientras que la segunda sí lo es. W. J. González, «Economic Prediction and Human Activity. An Analysis of prediction in Economics from Action Theory», Epistemologia, 17 (1994), pp. 205-246.

41 Este rasgo del sujeto humano como «sujeto de experiencias» se desarrolla en W. J. González, «La primitividad lógica del concepto de persona», Anales de Filosofia, 1 (1983), pp. 79-118. 
carencias como especie: el dominio científico es cognoscitivo, mientras que el tecnológico es transformador 42 .

Generalmente, como apunta Niiniluoto, la Tecnología tiene una carga ética mayor que la Ciencia, ya que tiene un más amplio margen de libertad, puesto que la producción de realidades artificiales permite seguir varios caminos alternativos $^{43}$. Ahora bien, en este punto cabría preguntar ¿qué es más artificial: un artefacto o un modelo teórico de la realidad? Está claro que la incidencia del artefacto sobre la realidad es mucho más directa que la del modelo teórico, pero ¿cuál es más determinante en el cambio de la concepción del mundo? A este respecto, las aplicaciones inmediatas que un artefacto puede tener no deben hacernos olvidar, a mi entender, las aplicaciones remotas que cabe dar sobre la realidad a partir de una determinada concepción del mundo. Estas últimas aplicaciones suelen ser mucho más importantes y duraderas que las aplicaciones de los artefactos. Un ejemplo lo tenemos en los CFCs (clorofluorocarbonados), ¿el error se produjo desde la Tecnología que los fabricaba y los ofrecía al uso masivo, o el fallo provenía, más bien, de una falta de investigación básica sobre tales compuestos que no advirtió respecto a la vida -hasta 50 años en la atmósfera- de sus moléculas?

En las relaciones entre la Ciencia y la Ética, como partes integrantes de la cultural, conviene tener en cuenta concepciones de cultura posteriores a la de Tylor, como puede ser la propuesta por Clifford Geertz, que parte de una idea central: «el hombre es un animal inserto en tramas de significación que él mismo ha tejido» 44 . Así, la cultura es «esa urdimbre (tejida por el ser humano) y que el análisis de la cultura ha de ser por tanto, no una ciencia experimental en busca de leyes, sino una ciencia interpretativa en busca de significaciones» ${ }^{45}$. Desde esta perspectiva, la Ética y la Ciencia, en cuanto lenguajes, son claros ejemplos de esas manifestaciones significativas de las que el ser humano se autoprovee.

Dentro del complejo cultural, la Ciencia ha pasado a ocupar desde hace algún tiempo uno de los puestos más relevantes. En la cultura occidental, que es la dominante en muchos aspectos -en especial, en el plano científico-, no resulta exagerado mantener, en algunos casos, la afirmación de P. K. Feyerabend sobre el carácter de la Ciencia como gran mito del siglo $X X^{46}$. Las dimensiones que la investigación científica ha ido adquiriendo durante los últimos dos

42 I. Niiniluoto, «Los límites de la Tecnología», pp. 402-403.

43 Ibid. , p. 403.

44 C. Geertz, La interpretación de las culturas. Barcelona: Gedisa, 1989, p. 20.

45 Ibid.

46 P. K. Feyerabend, «El mito de la ciencia y su papel en la sociedad», Teorema (1979), pp. 11-36. 
siglos ha hecho que sea necesario ir delimitando cada día más el imbricado edificio de la Ciencia y la Tecnología. Las Políticas científicas y tecnológicas (las Políticas de Investigación y Desarrollo, I+D) de los Estados occidentales han hecho que, desde el punto de vista financiero, sean muy relevantes los fondos destinados a la investigación, sobre todo aquellos destinados a investigaciones que tienen como fin inmediato la creación de herramientas eficaces para el dominio más completo del medio, tanto natural como social.

La fuerte repercusión que tienen los avances tecnológicos sobre la vida individual y social -debido, en parte, a la velocidad con la que se producen estos logros por la cantidad de apoyo financiero con el que cuentan-muestra la necesidad de realizar reflexiones de carácter ético sobre los distintos campos de la investigación científica y tecnológica. Entre estas consecuencias figura el cambio de nuestra concepción del mundo 47 , que está haciendo, por ejemplo, que consideremos como un fin social prioritario destinar fondos a determinadas áreas de investigación con vistas a una futura mejora de la calidad de vida. Aparentemente se abandonan metas y mejoras a corto plazo ante la conveniencia objetiva -y bien justificada desde el punto de vista racional- de abordar otras investigaciones a largo plazo, más beneficiosas a priori, pero sobre las que obra, como sobre toda materia de predicción, un alto porcentaje de incertidumbre acerca de sus futuras bondades reales y prácticas. Ahora bien, hoy parece claro que aquellos proyectos de investigación que no dan frutos en un plazo relativamente corto de tiempo o que dejan de estar en el interés de los subvencionadores ven disminuida su financiación o, sencillamente, eliminada.

\section{LA CIENCIA BÁSICA Y LAS VALORACIONES ÉTICAS}

La distinción entre Ciencia y Tecnología, así como la existencia de un campo intermedio -la Ciencia aplicada- resulta necesaria desde diversos puntos de vista, como el epistemológico o el metodológico. Sin embargo, aunque mantener la distinción puede ayudar a desentrañar cómo (o, más exactamente, hasta dónde) repercuten las consideraciones éticas en cada una de estas parcelas, puede también tener el efecto contrario, pudiendo conducir fácilmente a la creencia en la impunidad de determinadas parcelas, principalmente la correspondiente a la Ciencia básica. La confusión provendría, tal como se señaló en el segundo apartado, de dejar a la Ciencia al margen de las consideraciones éticas de carácter externo, por su carencia de consecuencias, y de las internas, pues se le supone que se cumplen en un grado óptimo, y a la Tecnología hacerla objeto de las demandas éticas externas por las consecuencias de sus desarrollos.

47 J. Ladrière, El reto de la racionalidad. Salamanca: Sígueme, 1977, pp. 95-97. 
Dejar caer la Ciencia básica fuera del ámbito de los juicios éticos externos sería, a mi entender, un error. Porque, tal como señala N. Rescher ${ }^{48}$, hay métodos de adquisición del conocimiento que no son éticamente aprobables sino todo lo contrario. $Y$ es en este punto en el que, a mi juicio, cabe equiparar la Ciencia y la Tecnología, es decir, en cuanto que actividades humanas ${ }^{49}$, y, por tanto, son susceptibles de valoraciones éticas. En este caso concreto, el conocimiento científico -que es un componente crucial en esa actividad-es, además, un tipo de conocimiento humano entre todos los demás posibles y, en concreto, entre los que de hecho se dan 50 .

$\mathrm{Si}$ admitimos, como propone Agazzi, que se puede establecer una finalidad a la Ciencia, independiente de los propósitos de los científicos, a saber: la adquisición de saber 51 , entonces dejaremos a la Ciencia básica al margen de toda consideración ética, pues no consideraremos las intenciones de los científicos como individuos, cayendo en la postura extrema, que cabe dentro de lo que Rescher llama el Laissez faire (el dejar hacer). Si, por el contrario, en el caso de la Tecnología, en cuanto Ciencia aplicada, sólo se puede aspirar a «proporcionar conocimientos eficaces encaminados a encontrar soluciones a cualquier problema concreto» 52 , cifraremos la bondad o no de un artefacto en función de su eficacia, lo que aparta la consideración ética tanto de la Tecnología en sí como de los tecnólogos como individuos. Ambas posturas conllevarían, a su vez, la obligación de liberar a «la industria del conocimiento en todos sus componentes de todas y cada una de sus regulaciones y controles» 53 .

Ahora bien, en cuanto que son partes integrantes de la cultura, la Ciencia y la Tecnología tienen carácter social, es decir, se llevan a cabo de manera colectiva y no individual, lo que hace difícil dejar a un lado las intenciones de los científicos y los tecnólogos como individuos, porque multiplica las posibles influencias externas (influencias que aumentan según el número de individuos que participan de acuerdo con una relación progresiva-exponencial, y por la disparidad de influencias que cada uno de ellos recibe).

Esta característica de la Ciencia y la Tecnología -ser una actividad colectiva- puede conducir a pensar que las responsabilidades quedan diluidas en el grupo. No obstante, el hecho de que las empresas científicas necesiten un gran número de investigadores $\mathrm{y}$, además, un gasto importante, conlleva la necesidad de financiación por parte de la sociedad, tanto de manera pública como

\footnotetext{
48 N. Rescher, Razón y valores en la era científico-tecnológica, cap. 8.

49 E. Agazzi, op. cit., pp. 230-232.

50 N. Rescher, The Limits of Science, pp. 208-215.

51 E. Agazzi, op, cit, p. 237.

52 lbid.

53 N. Rescher, Razón y valores en la era científico-tecnológica, cap. 8.
} 
privada. Esto, a su vez, conduce a que la sociedad se implique en el quehacer científico y que esté de alguna manera legitimada para supervisar el trabajo realizado. Por eso, a juicio de Martinez-Freire, la sociedad debe controlar y exigir responsabilidades éticas a la Ciencia-en concreto a los científicos que desarrollan la investigación para la que se obtuvo la financiación-, a través de unas comisiones mixtas que sean renovables, compuestas por ciudadanos ajenos a la Ciencia y por científicos ${ }^{54}$. Obviamente, todo lo anterior se refiere a la intencionalidad de los científicos cuando realizan una investigación determinada, pero también cabe hablar de las consecuencias de dicha investigación, para las que no valen argumentos como el ya citado de la financiación. Así, será revisable éticamente por la sociedad cualquier avance científico o tecnológico que implique consecuencias directas sobre el conjunto de la sociedad y no sólo porque haya sido financiado socialmente (puede darse el caso de una investigación que se lleve a cabo sin subvenciones oficiales ni particulares, sino que sea iniciativa de los propios investigadores que la realizan).

Todo este problema relacionado con la demanda de responsabilidades éticas a la Ciencia básica -su aparente impunidad frente a la revisión ética interna desde la sociedad y su nula incidencia en cuestiones éticas por su falta de consecuencias empíricas- se presenta, a mi entender, cuando el conjunto de las cualidades que, a juicio de Agazzi, acompañan a la investigación científica básica, tales como los hábitos morales de honestidad intelectual, espíritu de sacrificio, orden y disciplina, perseverancia, disponibilidad para aceptar las críticas, espíritu de colaboración y humildad 55 , traspasan el plano de lo que la Ciencia básica puede asumir. Es decir, estas cualidades, si son atribuibles a algún elemento de la investigación científica, ése no puede ser otro que el propio científico.

Siguiendo las directrices de Agazzi, se personalizan una serie de cualidades que se atribuyen a la Ciencia, cuando sólo pueden recaer sobre un sujeto. Y es precisamente porque sólo pueden atribuirse a un sujeto por lo que existe una probabilidad lo suficientemente alta de que no se cumplan. Además, estos valores resaltados por Agazzi, sólo constituyen el subconjunto de los valores internos de la Ciencia en el conjunto de la Axiología de la investigación científica. De esta manera, si se cumpliese el caso hipotético en el que todo científico los cumpliera, no dejarían de ser sólo una parte del entramado de valores que afectan al sujeto que investiga. El estado idílico de unos científicos alejados por completo de influencias ajenas al entorno científico (e, incluso, a los propios intereses) es -a mi entender-utópico. Este hecho -que el investigador

54 P. Martínez-Freire, loc, cit, pp. 174-175.

55 E. Agazzi, op. cit., p. 239. 
pone fines propios ajenos que son a la investigación-comporta, a mi juicio, que tanto la Ciencia básica como la aplicada y la Tecnología tengan que verse atravesadas por todo tipo de revisiones éticas por el conjunto de la sociedad (tanto valoraciones de índole interna -las referidas a la intencionalidad del científico y del tecnólogo-; como de carácter externa: las relativas a las consecuencias de la investigación que realizan).

Ahora bien, tampoco se quiere aquí caer en la postura contraria, denominada por Rescher Panregulación, que limita el quehacer científico a la «necesidad de saber", siendo así que esta necesidad "condiciona cada una y todas las investigaciones apropiadas. Saber es poder y el uso del poder en una comunidad debería siempre estar controlado y regulado. Más aún, el conocimiento pertenece a esa especial categoría ética de cosas que requieren un tratamiento particular y debería estar regulada con singular rigor» 56 . La posición más ajustada a la realidad sería -a mi entender-el Middle-of-the Roadism (el centrismo u opción moderada), postura propuesta por Rescher, que consistiría en no «tratar el conocimiento como un caso especial de cosas de cierto tipo, sino simplemente como un bien entre otros. Su búsqueda debería, por consiguiente, estar sujeta a la misma clase general de condiciones limitadoras (socialmente motivada) a las que nosotros sometemos otros bienes y fines» 57 , que supondría, de alguna manera, la desmitificación, propuesta por Feyerabend, de los resultados obtenidos por la Ciencia y la Tecnología, «Todo vale».

Esta misma postura (la posición moderada) es la que, a mi juicio, Agazzi perfila en los Capítulos XII ( $\ll$ La responsabilidad de la ciencia en un planteamiento sistémico») y XIV («Una ética para la Ciencia y la Técnica») de su libro El bien, el mal y la ciencia. A lo largo de estos dos capítulos, el sistema científico es presentado como un sistema más entre todos los que configuran el medio ambiente global (universal), en el que éste recibe y emite influencias tan dispares como lo son los mismos sistemas que, según Agazzi, conforman la vida humana, tanto a nivel intrasocial (sistemas no sociales: físico, biológico, ecológico, ...) y sociales (político, militar, religioso, ...) como desde una perspectiva extrasocial (sistemas científicos internacionales, sistemas internacionales no sociales y sistemas internacionales sociales no científicos) ${ }^{58}$. El proceso de retroalimentación (en forma de input y output) que se produce entre todos estos sistemas, da una medida más ajustada, a mi juicio, de lo que ocurre en realidad con la Ciencia y con la Tecnología que lo propuesto en el planteamiento de Agazzi, en el que quedan ambas dentro del sistema científico genéricamente. De esta manera, al igual que la Ciencia se ve influida por los restan-

56 N. Rescher, Razón y valores en la era científico-tecnológica, cap. 8.

57 Ibid.

58 E. Agazzi, op. cit., p. 313. 
tes sistemas, también la Tecnología influye y se ve influida por los restantes sistemas (salvando las distancias que pueden separarla teórica y metodológicamente de la Ciencia). Saliendo de la terminología de Agazzi y manteniendo que Ciencia, Tecnología y Etica son elementos culturales (no sistemas, como propone Agazzi), las relaciones entre estos tres ámbitos se vuelven aún más estrechas, por ser partes del todo que es la cultura.

Así pues, cuando en lo relacionado con las posibles responsabilidades éticas se defiende la existencia de diferencias entre la Ciencia y la Tecnología, había que señalar-como hace W. J. González- que «cada vez más, la Filosofía de la Tecnología se va configurando como un saber atento a una gama temática afín a la que se aborda en la Filosofía de la Ciencia, con las variaciones propias del lenguaje, sistema, conocimiento, método, realidad, objetivos y valores de la Tecnología»59. Este intento de dar fundamentos a la Tecnología y de proveerla de una base teórica similar a la correspondiente a la que avala a la Ciencia es ya una clara consecuencia que se podía vislumbrar desde el principio de este análisis. Así, la búsqueda del posible fundamento teórico de la Tecnología se puede estar convirtiendo en el refugio que los tecnólogos están reclamando para su parcela, una esfera donde puedan estar a salvo de los ataques y de las responsabilidades éticas. Y, aunque es cierto que a la Tecnología se le pueden pedir unas responsabilidades ética inmediatas en lo relativo a sus consecuencias, pues tienen una repercusión directa en la realidad y en los seres humanos, la Ciencia básica también las produce, tal vez de modo más recóndito, en términos de aumento de conocimiento; y esto puede tener repercusiones, por ejemplo, en el cambio de concepción del mundo que se puede operar en nosotros. Esto constituye una consecuencia externa al propio quehacer científico.

Al planteamiento que defiende el alejamiento de la Ciencia básica de las consideraciones éticas se puede añadir una dificultad: pretender que la conquista de los avances científicos básicos (por ejemplo, los nuevos conocimientos) puedan estar al margen de las valoraciones morales o de los juicios de valor sería como admitir que son los conocimientos quienes asaltan y se imponen a los científicos, quienes están ahí sólo como receptores pasivos. Desde esta perspectiva se olvidaría que el científico busca el conocimiento por el conocimiento, pero cada uno busca y encuentra unos determinados conocimientos y no otros que, en principio, también están a su alcance. El conocimiento está, así, modulado por el interés, consecuentemente, la experiencia científica no es puramente pasiva. Esta búsqueda selectiva marca una intencionalidad en el científico muy definida, que proviene de sus intereses personales, que están marcados, a su vez, por una multitud de condicionantes

59 W. J. González, «Progreso científico e innovación tecnológica: la 'Tecnociencia' y el problema de las relaciones entre Filosofía de la Ciencia y Filosofía de la Tecnología», p. 267. 
externos. Y, aunque el conocimiento en sí mismo no parece que pueda ser calificable de bueno o malo, sí puede serlo el orden y la prioridad con que se los haya buscado intencionalmente (por ejemplo, concocer los fundamentos teóricos de la energía nuclear en medio de una guerra mundial sería calificable por muchos de malo, aunque el conocimiento de la energía nuclear no sea algo malo en sí mismo e, incluso, pueda ser bueno en muchas ocasiones).

\section{CONSIDERACIONES SOBRE LA ETICA DE LA CiENCIA}

En algunos casos, los soportes teóricos son el paso definitivo para que se den los avances prácticos de la Ciencia aplicada y de la Tecnología. Ahora bien, hay de hecho algunos casos en los que el proceso es el inverso; esto es, sucede en ocasiones que los avances teóricos son arrastrados o provocados por las anticipaciones de índole práctico que han sido elaboradas con anterioridad (por ejemplo, la Teoría de la Computación ha llegado mucho más tarde que los desarrollos prácticos -la construcción de ordenadores-). Proponer una serie de categorías del conocimiento dependientes unas respecto de otras -en el caso más común, el conocimiento práctico respecto del teórico-supone afirmar que el conocimiento es jeraquizable en lo relativo a valores -el conocimiento teórico es mejor o superior al práctico, del que este último depende siempre-. Esto supone el reinado de determinados tipos de conocimiento, aquéllos que, como decía Platón 60 , están por encima de los demás y que quedan fuera del alcance de la mayoría de los mortales (no se va a discutir aquí la accesibilidad de cualquier conocimiento a cualquier inteligencia, por lo que se presenta solamente la posibilidad de acceso a priori) 61 .

Resulta conveniente admitir que el conocimiento cuenta con dos polos: el objeto conocido y el sujeto cognoscente, que obran con un peso específico cada uno de ellos en el proceso de adquisición de conocimiento (sin entrar aquí en cuál de estos polos tiene un peso mayor en el proceso de adquisición de conocimiento. Así, obviamente se elimina la posibilidad de que el conocimiento caiga sólo sobre el objeto y que la adquisión del conocimiento pudiera ser aséptica subjetualmente -los conocimientos emergen desde los objetos y se imponen a los sujetos-, único caso en el que podría admitirse una exención en cuanto a responsabilidades éticas del científico). No podemos más que asumir que, por parte del objeto, el conocimiento está dentro de los límites de la Naturaleza y que el sujeto también está dentro de la Naturaleza, pero con una parte

60 Platón, Diálogos, vol. IV: República, tr. C. Eggers Lan. Madrid: Gredos, 1992.

61 J. M. Martínez Selva, «Psicología del descubrimiento científico», en W, J. González (ed.), Aspectos metodológicos de la investigación científica. Madrid-Murcia: Ediciones Universidad Autónoma de Madrid y Publicaciones Universidad de Murcia, 1990, $2^{\text {a }}$ ed., pp. 305-315. 
que podríamos denominar «no objetivable»: su mente. Objeto y sujeto no sobrepasan juntos el ámbito natural (Naturaleza) y el humano (cultura), pero ¿cómo podemos decir que un conocimiento es superior a otro desde el análisis ético? $\mathrm{O}$, más aún, ¿cómo podemos trazar una línea en el terreno cognoscitivo que separe nítidamente lo que cae bajo el ámbito de la evaluación moral y lo que puede quedar fuera?

Estamos acostumbrados a escuchar que sólo los actos voluntarios de los seres humanos pueden caer bajo valoraciones de índole moral. También asumimos de manera generalizada que sólo los actos instintivos, como comer, respirar, etc., los que compartimos con los animales, son los únicos que pueden salvarse de la consideración moral. No podemos, sin embargo, eludir la responsabilidad en casos en los que no entren en juego ninguno de los condicionantes denominados «instintivos», que son todos los demás, incluso la búsqueda del conocimiento, que pasa a formar parte de los actos voluntarios del ser humano. Esto conlleva la intencionalidad del sujeto que realiza la investigación y, a su vez, que la investigación se vea orientada en un sentido, precisamente el que el investigador quiere darle.

Cualquier indagación viene motivada por alguna necesidad, por muy básica o teórica que ésta sea; y el investigador, que es quien indaga, está guiado por un propósito o finalidad. Y la posesión del conocimiento, aunque éste no vaya a tener una aplicación práctica directa, puede llegar a ser un arma tan efectiva o más en algunos casos que la aplicación misma de esos conocimientos. La Ciencia básica, como actividad voluntaria, difícilmente puede funcionar como una estructura totalmente independiente de los sujetos que la realizan; por lo tanto, no puede quedar al margen de las consideraciones éticas o morales. Porque, cuando el científico básico se propone alcanzar el conocimiento, esa tarea tenía, al menos, una finalidad práctica clara, que es un propósito del investigador: cubrir una necesidad de conocimiento personal; lo que es una actividad totalmente voluntaria y nada aséptica, pues el conocimiento, aunque sea teórico, siempre concede algún poder. Y la otra posibilidad es que entre las pretensiones del investigador sólo entrase, de manera altruista, ayudar a la Humanidad (si cabe admitir expresarse en los términos más abstractos posibles), lo que, en última instancia, lleva a una intencionalidad personal «propósito» en palabras de Agazzi-muy definida 62.

Si cabe disculpar las actuaciones de los seres humanos, por considerar que hay algunas que, por teóricas, están alejadas de la consideración moral, sólo conseguiremos que la Tecnología quiera (y a eso estamos abocados cada día más) alcanzar el estatuto teórico que tienen otras parcelas, como la Ciencia, para poder, en aras de la índole teorética de sus actividades, hacer cualquier 
cosa (como está pasando con los clones). La solución a las consideraciones éticas no creo que deba venir, pues, de la mano de la separación ética de la Ciencia en «básica» y «aplicada» y la Tecnología, para hacer que las responsabilidades recaigan sobre la parcela denominada «aplicada» y sobre la Tecnología, y dejando que la Ciencia básica pueda llevarse a cabo libre de cualquier examen moral o juicio ético. La corriente ética debe ser la inversa, es decir, intentar que sea la Ciencia teórica la que se someta, también, a los juicios éticos tanto internos como externos.

Y esta conexión entre Ciencia y Tecnología, que permite el flujo ético, se hace patente en la misma dificultad que entraña establecer sus diferencias desde el plano cultural, donde se presentan como productos humanos de distinta índole, pero humanos en la misma medida. Si la reflexión ética se extiende, como se mantiene en este trabajo, a todos los ámbitos específicamente humanos, y se acepta que la Ciencia básica es la muestra más significativa de aquello que diferencia al ser humano de los restantes animales, por darse en ella los niveles más altos de abstracción, entonces ¿cómo iba a escapar a la consideración moral o ética -en sentido amplio-, ésta que es una de las más genuinas muestras de lo humano? Los actos que se llevan a cabo cuando se pretende realizar una investigación teórica entran de lleno en el plano específicamente humano, por estar dotados de una finalidad concreta, que surge del propio sujeto que investiga y que no provienen de otro tipo de necesidades que puede estar más o menos impuestas por las condiciones exteriores, como pueden ser la supervivencia en medios hostiles.

Pilar Beltrán Orenes es ayudante de Lógica y Filosofía de la Ciencia en la Universidad de La Coruña. Autora de «Semántica de las nociones matemáticas de 'prueba' y 'demostración'», en C. Martín Vide (ed.), Actas del VIII Congreso de Lenguajes naturales y Lenguajes formales. Barcelona: PPU, 1992, pp. 181-187, y «La Matemática de Lakatos: el papel de la prueba en la Metodología», en J. Arana (ed.), La Ciencia de los filosofos. Sevilla: Universidad de Sevilla, 1996, pp. 305-320.

Dirección postal: Facultad de Humanidades, Universidad de La Coruña, Campus de Esteiro, c/ Dr. Vázquez Cabrera, s/n, E-15403 Ferrol-La Coruña.

E-mail:pbeltran@udc.es 